Eiji Yumoto, M.D., Kiyofumi Gyo, M.D., and Naoaki Yanagihara, M.D.

\title{
Resection of Persistent
}

\section{Nasopharyngeal Carcinoma}

\begin{abstract}
Although radiotherapy has been generally accepted as the treatment of choice for nasopharyngeal carcinoma (NPC), NPC at the primary site is not always controlled by this therapy. We performed surgical treatment to eradicate such residual tumor through a transmandibular, transpterygoid approach, on four patients with local residual NPC after curative radiotherapy. Two patients survived with no evidence of disease for more than 6 years. The other two patients died of multiple metastases to the liver or bone at 4 years and at 6 months after treatment. However, the nasopharynx remained free of disease in these two patients. Although the number of patients in the present series is small, surgical treatment of local persistent tumor after radiotherapy was beneficial. The indication for this technique is tumor in the nasopharynx extending to the parapharyngeal space, not invading intracranially. The transmandibular, transpterygoid approach offers a wide operative field with minimal postoperative morbidity, making it possible to manage the internal carotid artery easily. (Skull Base Surgery, 4(2):59-64, 1994)
\end{abstract}

Most nasopharyngeal cancers (NPC) are histologically poorly differentiated squamous cell carcinomas and are therefore very sensitive to irradiation. Surgical routes to reach and to obtain a wide operative field of the nasopharynx, situated at the center of the bony structure of the face, are very few. Frequently, initial symptoms derived from NPC are neck mass, epistaxis, and hearing loss. When one of these symptoms appears, NPC has already invaded the adjacent organs. Because of these characteristics of NPC, radiotherapy is generally accepted as the treatment of choice. However, NPC has a relatively poor prognosis compared with carcinomas arising from other head and neck regions, with the disease-free 5-year survival rate reported as 29 to $47 \% .^{1-6}$

The primary lesion very often appears after radiotherapy, determined by posterior rhinoscopic or fiberscopic observation. However, sometimes this is not the case, as reported by Sham et al. 7 Thirty-four of 379 NPC patients that they treated showed local tumor recurrence within 3 months after the completion of radiotherapy. They concluded that the primary lesion persisted in the 34 patients throughout the course. We have utilized computed tomography (CT) since 1982 and, recently, mag- netic resonance imaging (MRI) for follow-up during and after radiotherapy. These methods provide a reliable means for imaging the precise extent of tumor invasion and for assessing the effects of treatment. The results showed that NPC did not always disappear after $60 \mathrm{~Gy}$ of irradiation, even when posterior rhinoscopic or flexible fiberscopic observation did not indicate residual tumor.

Eradication of tumor in the primary site and cervical lymph nodes is essential to prevent metastatic lesions in distant organs. Many cases of regional relapse in the neck lymph nodes are amenable to curative radical neck dissection. On the other hand, the prognosis of those whose tumor persisted or recurred in the primary site is generally very poor. Only 5 to $28 \%$ long-term control was achieved when they were treated again with irradiation. ${ }^{7-10}$ Therefore, we performed surgery to eradicate such residual tumor through a transmandibular, transpterygoid approach. This approach is indicated for NPC extending to the parapharyngeal space. This article describes the operative procedure performed via the transmandibular, transpterygoid approach to the nasopharynx, and reports our experience of four NPC patients in whom persistent local tumor after radiotherapy was surgically resected. 


\section{PATIENT SELECTION}

The primary lesion was treated with a fractionated cobalt apparatus. Irradiation with parallel opposed lateral portals covered the primary nasopharyngeal lesion, base of skull, and retropharyngeal nodes. The whole neck was also irradiated regardless of the presence or absence of cervical lymph nodes. In addition, combined chemotherapy has been administered since 1985 . Two to 4 weeks after the completion of this treatment battery, each patient underwent CT or MRI examination. The results showed residual tumor in the primary site of 8 of 19 patients treated between 1982 and 1991. The extent of tumor in four of these eight was limited to the nasopharynx and parapharyngeal space, and therefore the tumor was resected through a transmandibular, transpterygoid approach. Operation at the primary site is not indicated in cases of intracranial extension, involvement of the internal carotid artery (ICA), or invasion into the sphenoid sinus, clivus, or vertebral bodies. Table 1 summarizes the age, sex, histologic results, and TNM classification based on that of the 1987 International Union Against Cancer. Histologic findings of four patients were squamous cell carcinomas, one of them being moderately differentiated and the other three poorly differentiated.

Table 1. Patient's Age, Sex, Histologic Findings, and Extent of Tumor

\begin{tabular}{ccccc}
\hline Patient & $\begin{array}{c}\text { Age } \\
(y r)\end{array}$ & Sex & $\begin{array}{c}\text { Histologic } \\
\text { Findings }\end{array}$ & $\mathrm{TN}^{+}$ \\
\hline 1 & 42 & $\mathrm{M}$ & SCC* & $\mathrm{T} 2 \mathrm{~N} 2 \mathrm{C}$ \\
2 & 68 & $\mathrm{M}$ & SCC & $\mathrm{T} 3 \mathrm{~N}^{*}$ \\
3 & 41 & $\mathrm{M}$ & SCC & $\mathrm{T} 3 \mathrm{~N} 2 \mathrm{C}$ \\
4 & 40 & $\mathrm{M}$ & SCC & T2N2c \\
\hline
\end{tabular}

*SCC: squamous cell carcinoma.

+TN classification is based on the 1987 International Union Against Cancer classification. A lesion metastatic to distant organs was not found in all patients when radiotherapy started.

\section{SURGICAL PROCEDURE}

Figure 1 illustrates the anatomic relationship of the upper parapharyngeal space and nasopharynx. The arrow in Figure 1 shows the direction of this approach. Temporary tracheostomy is necessary for maintaining an airway during the immediate postoperative period. A skin incision divides the lower lip in the midline and continues down through the chin. The incision then curves downward across the neck to just beneath the auricle, as shown in Figure 2, left. A mucosal incision is made from the corner of the lip incision, proceeding posteriorly along the lower gingivolabial sulcus. The incision crosses the ascending ramus of the mandible and ends close to the pterygoid hamulus (Fig. 2, center). A vertical skin incision may be added when neck dissection is necessary. Soft tissues, including skin, the masseter muscle, and parotid gland, are elevated from the mandible and retracted upward. This step preserves the facial nerve function completely. The mandible is severed just posterior to the third molar tooth. The ascending ramus of the mandible is then retracted upward. Resection of the coronoid process greatly facilitates mobility of the ascending ramus and helps prevent postoperative trismus. The exposed lateral and medial pterygoid muscles are divided in order to reach the lateral wall of the nasopharynx (Fig. 2, right). Identification of the large vessels in the upper neck and following them upward to the skull base are key points in this operation. As noted previously, when the ICA is involved, surgical treatment is not indicated. Without firm adhesion of NPC to ICA, blunt dissection of the vessel from NPC is not difficult. Retropharyngeal lymph nodes are usually dissected.

A small mucosal defect of the nasopharynx after tumor removal may be left without epithelial lining. Dead space left after large tumor resection is filled with the pterygoid muscles or a pedicled sternocleidomastoid muscle. ICA is protected by these muscles. The retracted ascending ramus assumes its original position and is fixed

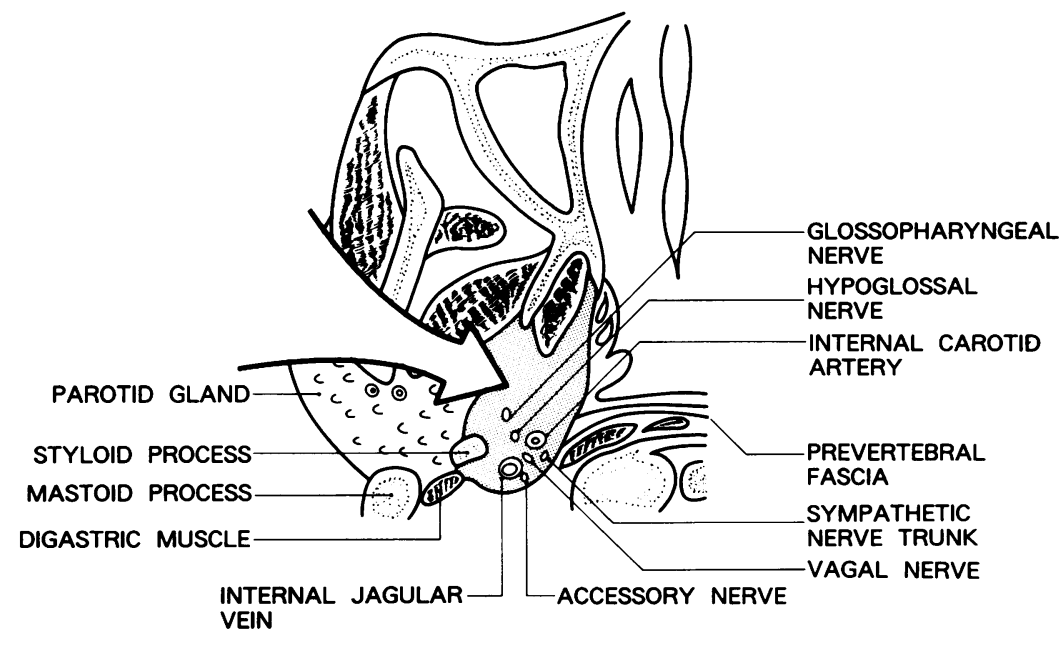

Figure 1. Anatomy of the parapharyngeal space (dotted area) and nasopharynx. An arrow indicates direction of the transmandibular, transpterygoid approach. 
Figure 2. Schematic drawing of surgical procedure. Left: Skin incisions. Center: Incision of oral mucosa. Right: Approach to the nasopharynx is made by retracting the ascending ramus of the mandible
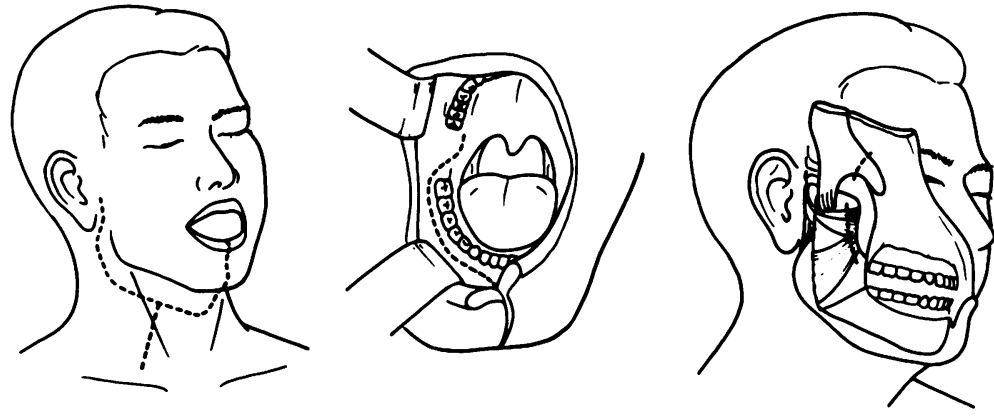
and soft tissue upward.

by a miniplate system. The tracheostoma is closed several days after the operation.

\section{CASE REPORTS}

\section{Patient 1}

A 42-year-old man developed a painless tumor in the right neck and underwent surgical removal elsewhere 2 years previously. In October 1984, he came to our hospital complaining of a left neck mass and was found to have NPC. Sixty Gy cobalt irradiation was given over 6 weeks (November 13 to December 25, 1984). Because CT examination suggested local persistence of tumor, it was surgically removed on February 5, 1985, through the transmandibular, transpterygoid approach. The patient's course had been uneventful until January 1989, when metastasis to the lumbar vertebrae occurred. He died 4 months later.

\section{Patient 2}

A 68-year-old man had occasional bloody sputum for half a year and was diagnosed as having NPC in November 1984. The right lateral wall of the nasopharynx bulged into the cavity and CT showed tumor invasion into the parapharyngeal space (Fig. 3, left). Radiotherapy was given in the period between December 3, 1984, and January 23,1985 . Two weeks after $60 \mathrm{~Gy}$ of irradiation, CT clearly revealed residual tumor extending from the nasopharynx to the parapharyngeal space (Fig. 3, center). Surgical tumor removal was performed 5 weeks after irradiation. The tumor lay posterior and superior to the opening of the eustachian tube and adjoined the ICA (Fig. 4). It was removed en bloc. The patient had been free of disease for 91 months since then and was recently lost to follow-up. Figure 3 (right) shows a CT image 5 months following the operation.

\section{Patient 3}

A 41-year-old man underwent right radical neck dissection elsewhere because of painless mass in the neck. Because histologic examination demonstrated squamous cell carcinoma, he was referred to us in January 1985. NPC was shown in a biopsy specimen taken from the nasopharynx. Radiotherapy was given in the period between February 19 and April 8, 1985. Two months after administration of radiotherapy combined with cisplatin chemotherapy, CT showed local residual tumor. It was surgically removed on June 25,1985 . Bone scintigram examined 1 month after the operation showed abnormal accumulation in the ishial bone. Although the nasopharynx remained free of disease, he died of multiple distant metastases to bone 6 months after the operation.
Figure 3. Computed tomograms of patient 2. Left: At the initial visit (before irradiation). Center: Two weeks after $60 \mathrm{~Gy}$ irradiation. Tumor did not disappear. Right: No evidence of residual tumor 5 months after operation.

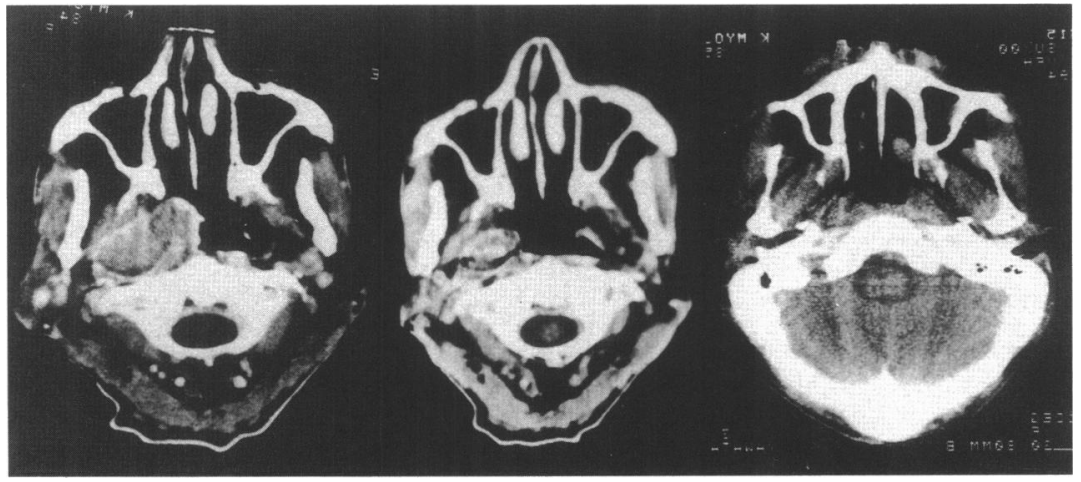




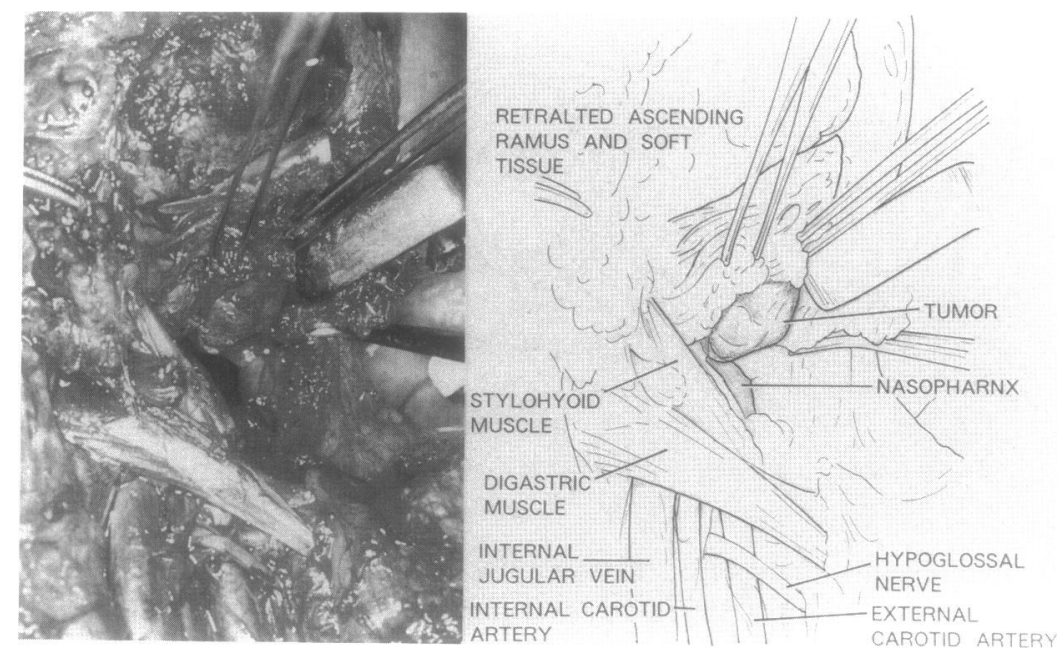

Figure 4. Operative view (left) and schematic illustration (right) of patient 2. Tumor lies posterior and superior to the opening of the eustachian tube and adjoins the internal carotid artery.

\section{Patient 4}

A 40-year-old man had bilateral serous otitis media for 2 months. Subsequently, he developed a painless neck mass on both sides and consulted our clinic in June 1986. He was diagnosed as having NPC and was given $60 \mathrm{~Gy}$ cobalt irradiation. On August 18, 1986, when radiotherapy was finished, fiberscopic observation showed an apparent tumor mass in the nasopharynx. Two weeks later, NPC was not fiberscopically identifiable, but CT suggested possible residual tumor. On September 9, 1986, we operated on the patient through a transmandibular, transpterygoid approach together with bilateral neck dissection. Histologic examination demonstrated viable tumor cells in the bilateral neck lymph nodes, but not in the specimen resected from the nasopharynx. The patient's course has subsequently been uneventful for 78 months.

\section{RESULTS}

Patients 2 and 4 survived with no evidence of disease for 91 and 78 months, respectively. Patients 1 and 3 died of multiple metastases to the liver or bone 4 years and 6 months after treatment, respectively. These two patients with distant metastases (1 and 3 ) had no tumor in the primary site, however.

Table 2 lists the intraoperative blood loss and post-

Table 2. Intraoperative Blood Loss and Postoperative Sequela

\begin{tabular}{ccl}
\multicolumn{3}{c}{ Sequela } \\
Patient & $\begin{array}{c}\text { Blood Loss } \\
(\boldsymbol{m})\end{array}$ & \multicolumn{1}{c}{ Sequela } \\
\hline 1 & 500 & Paresis of the soft palate \\
2 & 550 & None \\
3 & 1070 & None \\
4 & 850 & Infection of the mandible \\
\hline
\end{tabular}

operative sequelae of all cases. Blood loss ranged from 500 to $1070 \mathrm{~mL}$, with a mean of $740 \mathrm{~mL}$. Patients 2 and 3 did not have any sequelae after the operation. Patient 1 showed paresis of the soft palate, which improved later, but not completely. Slight nasality of speech was his only symptom. Infection of the mandible at the temporarily resected site occurred 2 months after the operation in patient 4 . Conservative treatment with pus drainage and local antibiotics failed to control the infection. The infected bone and miniplate were removed surgically, allowing the wound to heal. All patients except patient 4 were able to open the mouth sufficiently to eat.

\section{COMMENTS}

Although radiotherapy has been generally accepted as the treatment of choice for NPC, NPC at the primary site is not always controlled by this therapy. Increasing the local tumor control rate and decreasing the incidence of distant metastases are fundamental in treating NPC. EnPee et $\mathrm{al}^{4}$ reported that routine boosting of the dose to a residual lesion at the end of conventional radiotherapy failed to improve the prognosis of patients. Tsuji et $\mathrm{al}^{5}$ reported that adjuvant chemotherapy following radiotherapy lowered overall relapse rates both inside and outside the irradiated fields. However, their follow-up periods were shorter than 5 years, and, thus, their results are not conclusive. Al-Sarraf et al ${ }^{11}$ reported that combined radiotherapy and chemotherapy helped to reduce systemic metastases, but did not reduce the local control rate. On the other hand, Fisch ${ }^{12}$ recommended combined treatment of irradiation and surgery in order to raise the survival rate of NPC that fails to disappear after radiotherapy. We adopted surgical treatment for local residual tumor after radiotherapy in all patients except patient 3 , in whom surgery was undertaken after both radiotherapy and chemotherapy.

The nasopharynx and parapharyngeal space close to 
the skull base are relatively inaccessible without major postoperative complications. Fisch ${ }^{12}$ utilized an infratemporal fossa approach for six $\mathrm{T} 1$ or T2 patients, who were alive 2 and 5 years following surgery, and for seven T4 patients, resulting in suppression of trigeminal pain. Although this approach allows exposure of the nasopharynx from the lateral aspect, acquiring access is quite lengthy, as $\mathrm{Krespi}^{13}$ pointed out. In addition, hearing ability is always sacrificed postoperatively.

Tu et al, 9 Fee et al, ${ }^{10,14}$ and Van Hasselt ${ }^{15}$ adopted surgical removal of residual tumor at the primary site after radiotherapy. Their surgical routes to reach the nasopharynx were either transpalatal or lateral rhinotomy. These approaches do not offer a sufficiently wide operative field to manage large vessels easily. Krespi ${ }^{13}$ and Krespi and Sisson ${ }^{16}$ stated that the internal carotid artery should be kept in view during the skull base dissection. NPC extending to the parapharyngeal space can be removed by following the ICA from the neck to the skull base and resecting the structures medial to this vessel. By doing so, retropharyngeal lymph nodes are also dissected. However, with this transmandibular approach via midline mandibulotomy, the nasopharynx did not seem to be in a short distance to the surgeon's hands, although this approach provided a relatively wide operative field.

Wei et al ${ }^{17}$ proposed the maxillary swing approach to the nasopharynx, and resected persistent NPC after radiotherapy. According to their report, the ICA is palpable, but not observable under direct vision. Therefore, as they commented, the indication of this method is quite limited, being applicable to NPC confined to the immediate neighborhood, especially for small tumors. In addition, postoperative trismus is inevitable.

In 1966, Ross and Sukis ${ }^{18}$ first reported a "pterygoid route" to remove adenoid cystic carcinoma of the nasopharynx. Nessel and Mundnich ${ }^{19}$ described a similar technique in 1974 as "transpterygoid access" or "lateral nasopharyngostomy." We applied a basically similar technique to four NPC patients, and our experience proved that a transmandibular, transpterygoid approach offers a wide operative field, making it possible to easily manage large blood vessels. In addition, the operative field is accessible to the surgeon compared with the other approaches just mentioned. Other approaches may be combined with this approach, depending on the location and extent of the lesion.

Since the mandible is temporarily divided posterior to the third molar tooth, resulting in cutting of the inferior alveolar artery, the blood supply to the mandible is decreased. This may have contributed to the infection of the mandible that occurred 2 months after the operation in patient 4 , although hyperreactivity of the skin and mucous membrane due to irradiation was suspected to be the main cause. As Attia et al ${ }^{20}$ reported, temporary removal of the ascending ramus of the mandible without severing the inferior alveolar artery facilitates obtaining a wider operative field as well as maintaining blood flow to the mandible. Another postoperative sequela was paresis of the soft palate in patient 1 . The paresis subsequently improved and he did not have swallowing difficulty.

Although two of our patients (1 and 3) developed distant metastases, the nasopharynx remained free of disease in these patients. Since metastasis to the bone in patient 3 became evident only 1 month after the operation, it was considered to have occurred prior to the operation. Patients 2 and 4 have been well for more than 6 years. Histologic examination indicated viable malignant cells in patient 2. In patient 4, however, in spite of the existence of an area of enhancement in CT image suggesting local persistent disease, histologic examination of the specimen resected from the primary site failed to demonstrate viable malignant cells. Whether malignant cells survived locally or not was not confirmed because whole serial sections were not prepared. Sham et al ${ }^{7}$ recommended that the decision to treat persistent disease should be withheld unless positive biopsy samples are obtained 10 or more weeks following radiotherapy. We generally agree with their advice, and also believe that when CT or MRI clearly delineates a local persistent tumor mass 4 weeks after radiotherapy additional treatment is indispensable. Unfortunately, the tumor frequently invades intracranially, and, thus, only palliative treatment is indicated in the majority of such patients.

In conclusion, surgery is beneficial in a limited number of cases of radiation failure, as shown in this article. The transmandibular, transpterygoid approach offers a useful surgical route in the treatment of local persistent tumor in the nasopharynx and extending to the parapharyngeal space.

\section{REFERENCES}

1. Levendag PC, Huygen PLM, Kazem I, et al: Malignant tumor of the nasopharynx: Retrospective review of 74 cases. Clin Radiol 34:451-458, 1983

2. Dickson RI, Flores AD: Nasopharyngeal carcinoma: An evaluation of 134 patients treated between 1971-1980. Laryngoscope 95:276-283, 1985

3. Chatani M, Teshima T, Inoue $\mathbf{T}$, et al: Radiation therapy for nasopharyngeal carcinoma: Retrospective review of 105 patients based on a survey of Kansai Cancer Therapist Group. Cancer 57:2267-2271, 1986

4. En-Pee Z, Pei-Long C, Ying-Fu C, et al: Radiation therapy of nasopharyngeal carcinoma: Prognostic factors based on a 10 year follow-up of 1302 patients. Int J Radiat Oncol Biol Phys 16:301-305, 1989

5. Tsuji H, Kamada T, Tsuji $\mathbf{H}$, et al: Improved results in the treatment of nasopharyngeal carcinoma using combined radiotherapy and chemotherapy. Cancer 53:1668-1672, 1989

6. Bailet JW, Mark RJ, Abemayor E, et al: Nasopharyngeal carcinoma: treatment results with primary radiation therapy. Laryngoscope 102:965-972, 1992

7. Sham JST, Choy D, Wei WI, et al: Value of clinical follow-up for local nasopharyngeal carcinoma relapse. Head Neck 14:208217, 1992

8. Yan $\mathrm{JH}, \mathrm{Hu} \mathrm{YH}, \mathrm{Gu} \mathrm{XZ}$ : Radiation therapy of recurrent nasopharyngeal carcinoma. Acta Radiol Oncol 22:23-28, 1983

9. Tu G, Hu Y, Xu G, et al: Salvage surgery for nasopharyngeal carcinoma. Arch Otolaryngol Head Neck Surg 114:328-329, 1988

10. Fee WE Jr, Robertson JB, Goffinet DR: Long-term survival after 
surgical resection for recurrent nasopharyngeal cancer after radiotherapy failure. Arch Otolaryngol Head Neck Surg 117: 1233-1236, 1991

11. Al-Sarraf M, Pajak TF, Cooper JS, et al: Chemo-radiotherapy in patients with locally advanced nasopharyngeal carcinoma: A radiation therapy oncology group study. J Clin Oncol 8:13421351,1990

12. Fisch U: The infratemporal fossa approach for nasopharyngeal tumors. Laryngoscope 93:36-44, 1983

13. Krespi YP: Lateral skull base surgery for cancer. Laryngoscope 99:514-524, 1989

14. Fee WE Jr, Gilmer PA, Goffinet DR: Surgical management of recurrent nasopharyngeal carcinoma after radiation failure at the primary site. Laryngoscope 98:1220-1226, 1988
15. Van Hasselt CA: The role of operations in the treatment of nasopharyngeal carcinoma. Ear Nose Throat J 69:279-281, 1991

16. Krespi YP, Sisson GA: Skull base surgery in composite resection. Arch Otolaryngol 108:681-684, 1982

17. Wei WI, Lam KH, Sham JST: New approach to the nasopharynx: The maxillary swing approach. Head Neck 13:200-207, 1991

18. Ross DE, Sukis AE: Nasopharyngeal tumors: A new surgical approach. Am J Surg 111:524-530, 1966

19. Nessel E, Mundnich K: Chirurgie des Epipharynx. In: Naumann HH: Kopf- und Hals-Chirurgie, vol 2, part 2. Stuttgart: Georg Thieme, 1974, pp 667-670

20. Attia EL, Bentley KC, Head T, et al: A new external approach to the pterygomaxillary fossa and parapharyngeal space. Head Neck Surg 6:884-891, 1984

\section{REVIEWER'S COMMENTS}

Drs. Yumoto, Gyo, and Yanagihara are to be commended for utilizing mandibulotomy and mandibular swing for a lateral approach to persistent nasopharyngeal carcinoma following radiation therapy. The technique itself is not a new one, but the application of it is new and has several advantages to handling the problem of persistent or recurrent disease after radiation failure. The most attractive aspect of this approach is that it allows direct visualization of the internal carotid artery to its entrance into the foramen lacerum. A disadvantage of the technique is that it does not provide easy access to nasopharyngeal carcinoma entering into the nose, or to good visualization of the medial aspect of the internal carotid artery, which is most likely to be at risk for most patients with recurrent disease.

I have had the opportunity of operating on 26 patients with recurrent disease after one or two courses of radiation therapy via a transpalatal, transmaxillary, and transcervical approach. Although I have identified the internal carotid artery to the foramen lacerum in almost all cases, sadly, on my 19th case, I inadvertently cut the internal carotid artery at the base of the skull. Fortunately, the first two patients that I operated on are alive and well 9 years following their surgery and the overall survival for patients eligible for 5 -year follow-up has been $44 \%$. As the current article so well demonstrates, control of disease at the primary site is only part of the battle and distant metastases continue to be a major problem for many of these individuals. Thus, before surgery is undertaken, a computed tomography (CT) scan of the chest, measurement of liver enzymes, and alkaline phosphatase, and, if indicated, CT scan of the liver and bone scan should be performed to ensure that no distant metastases are present. Once that has been done, the choice of surgical technique utilized is dictated by the tumor presentation.

Because the disease presents in a diversified fashion, a diversified surgeon with a diversity of techniques will be indicated. Although one could excise the tumor shown in Figure 3 via a transmaxillary and transpalatal approach, the latter approach advocated by the authors combined with the transpalatal approach would provide, in my opinion, the best approach to resecting this tumor. The difficulties with only a lateral approach is that it does not provide easy and safe access to the opposite side of the nasopharynx, which occasionally has to be removed, especially in patients who have recurrent disease that is submucosal following radiation therapy.

The other major disadvantage of this technique is the mandibulotomy itself and the potential for infection, as occurred in patient 4 . Marginally viable bone following radiation therapy is only further compromised by mandibulotomy.

Willard E. Fee, Jr., M.D.

\section{AUTHORS' REPLY TO COMMENTS}

Before proceeding to an operation, we always confirmed the absence of any single distant metastatic lesion by examining Ga scintigraphy of the whole body in addition to clinical tests as cited by the reviewer. Furthermore, we always checked scc-related antigen, EB virus titer and the possibility of presence of another concurrent cancer in other part of the body. Although a metastatic lesion in the ishial bone became evident one month after the operation in patient 3 , bone scintigraphy performed just after completion of radiotherapy did not indicate such metastatic lesion. 